\title{
SEC-ICP-MS and on-line isotope dilution analysis for characterisa- tion and quantification of immunochemical assays
}

\author{
David Clases ${ }^{1} \ddagger$, Raquel Gonzalez de Vega ${ }^{1}+$, David Bishop ${ }^{1}$, Philip Doble ${ }^{1}$ \\ ${ }^{1}$ Elemental Bio-Imaging Facility, University of Technology Sydney, Sydney, NSW, Australia
}

\begin{abstract}
This study presents a novel method based on size exclusion chromatography-inductively coupled plasma-mass spectrometry (SEC-ICP-MS) for the characterisation and quantification of immunoassays employing lanthanide-labelled antibodies. The addition of oxygen into the dynamic reaction cell improved the detection of sulphur as a marker for the antibodies and target protein via mass-shifting $(\mathrm{LOD}=3.7 \mathrm{ng} / \mathrm{mL}$ ), whilst maintaining sufficient sensitivity for the analysis of the lanthanides. Ultra-high performance liquid chromatography (UHPLC) SEC ensured a rapid high-resolution chromatographic method with separation times under 7 minutes of the labelled and unlabelled antibodies, the immunocomplexes, and the unconjugated polymer used to lanthanide-label the antibodies. SEC calibration estimated the molecular weights of all peaks and provided new insights in immunochemical reactions and the stoichiometry of the reactants and products. A novel on-line isotope dilution analysis (IDA) enabled absolute quantification of sulphur and lanthanide signals and the protein of interest. The chromatographic separation of immunocomplexes and labelled antibodies allowed the simultaneous determination of the antibody/metal stoichiometry and target protein concentration from a single mass flow chromatogram. An immunoglobulin protein was quantified after incubation with an ${ }^{153} \mathrm{Eu}$ labelled primary polyclonal antibody. The procedure was validated with direct labelling of the target protein with ${ }^{156} \mathrm{Gd}$ for parallel, simultaneous quantification. The concentration determined via direct labelling of the protein deviated only $1.9 \%$ from the immunochemical approach employing ${ }^{153} \mathrm{Eu}$ labelled polyclonal antibodies.
\end{abstract}

\section{INTRODUCTION}

The function of many biological processes relies on the up and/or down regulation of specific proteins ${ }^{1,2}$. Fundamental understandings of such processes require accurate determination of protein levels. Immunochemical assays, where an antibody is applied to a sample to target a specific epitope on a biomolecule, are one of the most widely used techniques for protein analysis. Fluorescent labels with distinguishable spectral emission bands allow multiplexed analyses that detect several proteins in a single sample ${ }^{3,4}$. This is particularly important, as a change in biological response is often the result of changes in the expression of more than one protein. However, broad emission bands limit the number of antigens observable, combined with low dynamic calibration ranges and photobleaching effects result in poor reproducibility and often inaccurate quantification. Early efforts to quantify proteins included the use of radioisotope labelled antibodies ${ }^{5}$, leading to many emerging technologies for improved antibody detection and quantification by exploiting labels with specific chemical features. In 2001 Zhang et al. first introduced element-labelled antibodies for analysis by inductively coupled plasma-mass spectrometry (ICP-MS), a detector with high sensitivity and selectivity as well as with a large linear calibration range ${ }^{6}$. The labelling of antibodies with heteroatoms permits quantitative ICP-MS analysis ${ }^{7,8}$, whilst multiple antibodies with distinguishable isotopic tags may be used for simultaneous multiplexed analysis of up to 30 different immunoassays ${ }^{9}$. Polymer labels have been synthesised to offer numerous metal binding sites resulting in antibodies labelled with up to 200 atoms further enhancing sensitivity ${ }^{10-13}$.
Chemical modification of antibodies is generally accompanied by an alteration of the antibodies properties ${ }^{14}$. Such alterations can range from minor changes in conformation, up to fragmentation and loss of affinity toward the antigen. Antibodies consist of two main regions, the $\mathrm{F}_{\mathrm{c}}$ (fragment crystallisable region), and the $F_{a b}$ (fragment antigen binding region) which binds to the target epitope on the biomolecule. Most labelling protocols aim to modify the antibody in the $F_{c}$ region via reduction of disulphide bridges. The exposed free thiol groups can subsequently be conjugated to a linker molecule (e.g. maleimide) which is connected to the metal label ${ }^{15}$. Unfortunately, the reduction reactions are not entirely selective and may generate a mixture of antibody fragments and conjugates lacking affinity or metal label. Such mixtures result in a loss of sensitivity and also affect selectivity, robustness and reproducibility of the entire method. An on-site, parallel and rapid quality analysis is therefore required to monitor the integrity of the labelled antibody, the degree of labelling, and the antibody-antigen affinity. Hann et al. used immunoaffinity assisted size exclusion chromatography (SEC)-ICP-MS for the targeted analysis of proteins and peptides in complex biological matrices which resulted in improved selectivity and sensitivity ${ }^{16}$. Nevertheless, Hempen and Karst pointed out that the analysis of immunoassays with chromatographic methods is limited due technical difficulties associated with long equilibration times of the stationary phase ${ }^{17}$. Terenghi et al. published a method to separate different immunocomplexes from labelled antibodies which offered a foundation for quantification, as it may be used to account for unreacted antibody ${ }^{18}$. However, quantification is only possible if the stoichiometry between the metal and the antibody 
is known ${ }^{14,19}$. Mueller et al. described a SEC-ICP-MS method to characterise a MeCAT-labelled antibody ${ }^{20}$. They added oxygen to a dynamic reaction cell (DRC) to enable the mass shifting of $\mathrm{S}$ and lanthanides for the analysis of antibody fragments generated during the reduction of the disulphide bonds. Mairinger et al. employed a similar method and determined the labelling degree of antibody fragments by weighting the metal and sulphur signal of respective peaks with a response factor ${ }^{7}$. Recently, Perez et al. employed SEC-ICP-MS to quantify immunoassays of four cancer biomarkers employing monoclonal antibodies. They calculated the labelling degree by determining the metal content via ICP-MS and the antibody concentration via a colorimetric assay using BSA as calibration standard ${ }^{21}$.

Although elemental quantification by ICP-MS is straightforward, the determination of protein concentrations via metal labels attached to antibodies is far from simple as the number of metals per label, labels per antibody and antibodies per targeted protein must be taken into account for each immunoassay. When polyclonal antibodies are used for immunoassays, it has to be considered that the ratio of antibody to the targeted protein can exceed a value of one. Sophisticated approaches like LCICP-MS and ESI-MS/MS for external and internal standardisation have been presented for the quantification of proteins and are generally transferable to immunoassays ${ }^{8,22}$. Quantification may be simplified by isotope dilution analysis (IDA), a technique recognised by CCQM (Consultative Committee for Amount of Substance: Metrology in Chemistry and Biology) as a metrological method of the highest quality ${ }^{23}$. IDA involves the addition of an enriched isotopic spike to deliberately alter the natural abundances, and determining concentration from these differences ${ }^{24}$. Species un-specific IDA is a technique developed for LC-ICP-MS analysis, and has been used in immunochemical assays to determine post-column recovery rates ${ }^{8}$. Yan et al. labelled proteins and determined 100\% labelling efficiency via LC-ICP-IDA-MS ${ }^{25}$. Unfortunately, this approach is not suited for the analysis of antibodies as complete labelling was accompanied with a loss of structural integrity.

Here, we developed an ultra-high performance SEC-IDAICP-MS method for a fast and robust quality analysis of antibodies labelled with MAXPAR ${ }^{\circledR}$ conjugation kits. Oxygen was added to a DRC to enable the analysis of sulphur in the antibodies, and a double isotope dilution analysis was used to characterise and relate the sulphur and metal content for quantification of the labelled antibody and analyte.

\section{MATERIALS AND METHODS}

\section{Chemicals and Consumables}

High purity ICP-MS standard calibration solutions of $10 \mathrm{mg} \mathrm{L}^{-1}$ dysprosium, europium, gadolinium and sulphur were purchased from Choice Analytical (Thornleigh NSW, Australia). Enriched ${ }^{34} \mathrm{~S}$ was obtained from ISC Science (Oviedo, Spain). The concentration of sulphur in this solution was determined by reverse isotope dilution analysis with a certified standard containing sulphur in natural abundance. Ammonium acetate (99.999\% purity) used to prepare the SEC eluent was purchased from Sigma Aldrich (Castle Hill, NSW, Australia).

Rabbit control IgG was obtained from R\&D systems (Minneapolis, USA). A primary goat anti-rabbit polyclonal antibody and a secondary donkey anti-goat polyclonal antibody were purchased from Sigma Aldrich. The protein of interest as well as the primary and secondary antibody were labelled with ${ }^{153} \mathrm{Eu}$, ${ }^{156} \mathrm{Gd}$ and ${ }^{163} \mathrm{Dy}$, respectively, using the MAXPAR ${ }^{\circledR}$ antibody conjugation kits (Fluidigm; formerly DVS Sciences, San Francisco, CA) according to the manufacturer's standard protocol (version 4 06/13).

\section{Instrumentation and Experimental Parameters}

SEC was performed using an ACQUITY UPLC SEC column $1.7 \mu \mathrm{m}, 4.6 \times 300 \mathrm{~mm}$ (Waters, Milford, MA, USA) on a Vanquish Flex Binary UPLC system (Thermo Fisher, Waltham, MA, USA) operated by Chromeleon Chomatography Management system (Thermo Fisher). An ammonium acetate buffer (0.1 M, pH 6.8) was used as mobile phase and the flow rate was optimised to $450 \mu \mathrm{L} / \mathrm{min}$. The SEC molecular weight calibration was performed with a protein standard containing thyroglobulin $(660 \mathrm{kDa}), \mathrm{V}$-globulin $(150 \mathrm{kDa})$, chicken albumin $(42.7 \mathrm{kDa})$ and ribonuclease $\mathrm{A}(13.7 \mathrm{kDa})$.

The chromatographic system was coupled to a Thermo iCAP RQ ICP-MS instrument (Thermo Fisher) which was operated in CCT-mode. Typical instrumental parameters are listed in Table 1. Oxygen was used as reaction gas (ultra-high purity, BOC, North Ryde, NSW, Australia) and the DRC parameters for mass-shifting ${ }^{34} \mathrm{~S}$ and ${ }^{32} \mathrm{~S}$ were optimised with a $100 \mathrm{ng} / \mathrm{mL}$ sulphur standard. ICP-MS dwell times were set to $10 \mathrm{~ms}$ to obtain sufficient data due to UHPLC conditions and a relatively large number of analytes. LOD and LOQ of sulphur were calculated according to the $3-\sigma$ and $10-\sigma$ criterion, respectively.

\section{Table 1. ICP-MS instrumental parameters.}

\begin{tabular}{ll}
\hline Tune parameter & Value \\
\hline CCT bias & $-6.2 \mathrm{~V}$ \\
D1 lens & $-349.4 \mathrm{~V}$ \\
CCT flow & $0.3 \mathrm{~mL} / \mathrm{min}$ \\
CCT entry lens & $-44 \mathrm{~V}$ \\
CCT focus lens & $1.5 \mathrm{~V}$ \\
CCT exit lens & $-70 \mathrm{~V}$ \\
Nebuliser flow & $1.11 \mathrm{~L} / \mathrm{min}$ \\
Plasma power & $1550 \mathrm{~W}$ \\
\hline
\end{tabular}

\section{Isotope Dilution Analysis}

The quantification of sulphur and lanthanide was performed by post-column isotope dilution analysis. A spike solution was continuously introduced (at $1.22 \mathrm{~mL} \mathrm{~min}^{-1}$ ) using a peristaltic pump (Gilson, Middleton, WI, USA) through a T piece. The acquisition of transient mass data of the spike solution was received via RS-232 and transformed into keystrokes with the software 232 key (Born, Luxembourg). The spike solution contained an isotope enriched ${ }^{34} \mathrm{~S}$ standard $(500 \mathrm{ng} / \mathrm{mL})$ for IDA of sulphur integral to antibodies and target protein, and Dy, Eu and Gd ICP-MS calibration standards $(5 \mathrm{ng} / \mathrm{g})$ for reverse IDA of the enriched MAXPAR ${ }^{\circledR}$ labels. The detector dead time was calculated according to Rodriguez-Gonzalez et al. ${ }^{24}$ and compensated by the acquisition software. Mass bias effects were considered by offline ICP-MS characterisation of the abundance of the natural and enriched isotopes. Concentrations were obtained following the integration of the mass flow signals using 
OriginPro 2017 software (version b9.42.380, Microcal Software Inc., Northampton, MA, USA). The target protein concentration was calculated based on the estimation that the number of sulphur atoms per IgG was 32 and the molecular mass $153 \mathrm{kDa}$.

\section{RESULTS AND DISCUSSION}

\section{Optimisation of ICP-MS parameters}

The sulphur content may be used to calculate the concentration of an antibody by knowledge of the antibody's primary structure, and the IgG subclass to provide the number of cysteine residues. The high abundance of spectral interferences on both sulphur isotopes limits the applicability of conventional ICP-MS in commercially available antibodies because the concentration of sulphur present is below typical LODs. These spectral interferences were avoided by addition of oxygen reaction gas in the DRC to form $\mathrm{SO}^{+}$adducts, resulting in improved LODs. Consideration was also given to potential mass shifts of the metal labelled to the antibody, as depending on the redox potential of the metal, it may itself undergo a mass-shift. Except for $\mathrm{Eu}$, all lanthanides formed mono-oxygenated species. Figure $1 \mathrm{a}$ ) shows the improvement in sensitivity and the reduction of background for the analysis of sulphur through mass-shifting to $\mathrm{SO}^{+}$. The LODs were determined to be 3.7 and $4.4 \mathrm{ng} / \mathrm{g}$ for ${ }^{32} \mathrm{~S}^{16} \mathrm{O}$ and ${ }^{34} \mathrm{~S}^{16} \mathrm{O}$, respectively. The analytical figures of merit are listed in Table 2. Except for Eu, the employment of a DRC decreased the signal obtained from the lanthanides and required the analysis of the mass-shifted product (see Figure $1 \mathrm{~b}$ ).
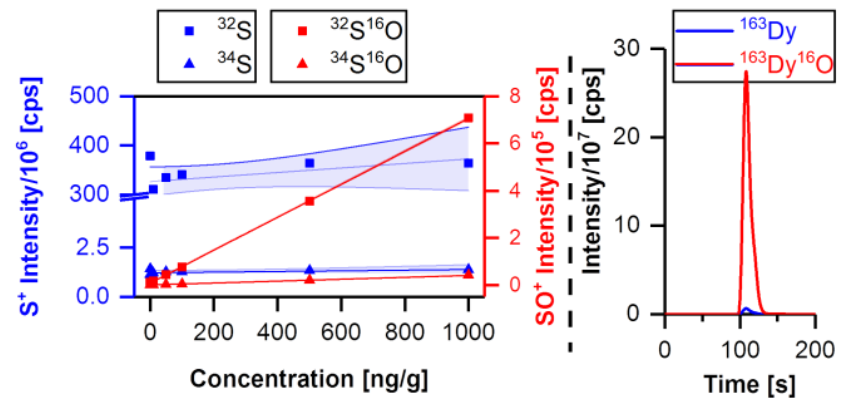

Figure 1. a) Improvement in sensitivity and background due to the employment of a DRC to mass-shift $\mathrm{S}^{+}$to $\mathrm{SO}^{+}$. b) The DRC favoured the formation of mono-oxygenated lanthanide adducts.

The elimination of the spectral interferences on ${ }^{32} \mathrm{~S}$ and ${ }^{34} \mathrm{~S}$ in the DRC provided selective detection of sulphur signals from labelled antibody solutions and immunoassay reagents. Antibodies are usually diluted in a stabiliser solution often comprising of high molecular weight sulphur-containing compounds that contribute to high backgrounds and produce confounding peaks during the analysis of antibodies via ICP-MS and SECICP-MS, respectively. Antibody solutions and immunoassay reagents should therefore be analysed without dilution with stabilising agents, or the stabiliser previously checked for interfering compounds.
Table 2. Figures of merit for the analysis of sulphur.

\begin{tabular}{lllll}
\hline Analyte & $\begin{array}{l}\text { Slope } \\
{[\text { cps g/ng] }}\end{array}$ & $\begin{array}{l}\text { Intercept } \\
{[\mathbf{c p s}]}\end{array}$ & $\mathbf{R}^{2}$ & $\begin{array}{l}\text { LOD } \\
\text { LOQ } \\
{[\mathbf{n g} / \mathbf{g}]}\end{array}$ \\
\hline${ }^{32} \mathrm{~S}^{16} \mathrm{O}$ & $699 \pm 2$ & $7910 \pm 903$ & 0.9999 & $\begin{array}{l}3.7 \\
12\end{array}$ \\
& & & & 4.4 \\
${ }^{34} \mathrm{~S}^{16} \mathrm{O}$ & $40.4 \pm 0.2$ & $577 \pm 84.6$ & 0.9998 & 15 \\
\hline
\end{tabular}

\section{Analysing labelled antibodies by SEC-ICP-MS}

SEC-ICP-MS has been used to monitor immunoassays ${ }^{21}$, however limitations in terms of long regeneration times ${ }^{17}$ or low chromatographic resolutions have prevented widespread uptake. This limitation was overcome by using a UHPLC instrument and column with high flowrates permitting rapid stationary phase regeneration and rapid sequential analyses, providing reproducible separation of the immunocomplexes, labelled antibodies, unlabelled antibodies and unconjugated polymer in less than 7 minutes with a flow rate of $450 \mu \mathrm{L} / \mathrm{min}$. These optimal conditions were applied to monitor the antibody-labelling reaction to identify the degree of labelling and the amount of metal per label.

The antibody reduction step and the product stability, which is influenced by storage conditions, pose ongoing challenges to the quantification of immunoassays. The reduction has a crucial impact on the degree of labelling as it may affect the antibodyantigen affinity and requires careful monitoring. Unsuitable conditions or long-term storage can result in undesirable reactions or conformational changes of the labelled antibody. Both were observable with SEC-ICP-MS through a change of retention time $\left(t_{R}\right)$, peak shape and peak symmetry. Figure 2 compares the SEC-ICP-MS chromatograms of a labelled antibody measured directly after labelling (red) and after four months storage (blue) in an un-stabilised solution. An unspecific reaction and/or conformational changes in the stored antibody was identified by a heavier or bulkier derivative observable as a shoulder detected at $t_{R}=290 \mathrm{~s}$. SEC-ICP-MS can therefore be employed to determine the integrity of stored antibodies by monitoring peak shape and retention. A further signal was observed at $t_{R}=415 \mathrm{~s}$ and was detected in all MAXPAR ${ }^{\circledR}$ labelled antibody solutions. The signal did not contain any sulphur, excluding its origin from the antibody (i.e. an antibody fragment). Spiking experiments with lanthanide-containing polymer identified the signal as the unconjugated loaded-polymer. The identification of the degree of unconjugated polymer label is important in immunohistochemistry assisted LA-ICP-MS as it is believed to cause non-specific background staining leading to decreased contrast and false positive results ${ }^{14}$. Substantial fragmentation of the antibody detected by other groups and reviewed by Kretschy et al. ${ }^{26}$ was not found in the framework of this study. 


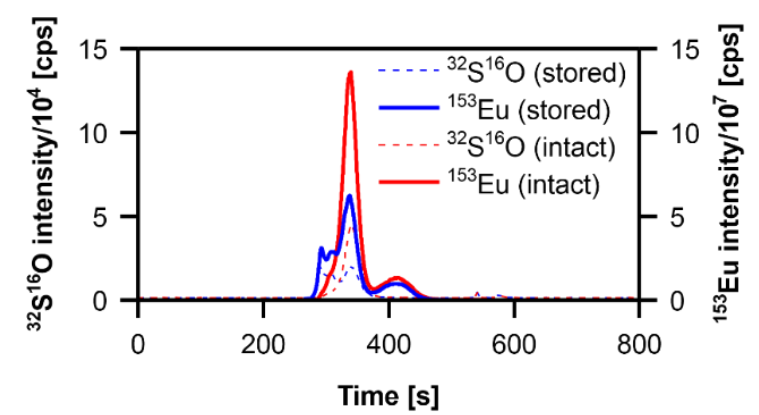

Figure 2. Comparison of peak shape and intensity of the same ${ }^{153}$ Eu-labelled antibody batch before (red line) and after long-term (4 months) storage (blue line).

\section{Analysing immunoassays with SEC-ICP-MS}

As the retention time in SEC is a function of its size, differences in elution time of labelled and unlabelled antibody, or the label alone, can be used to estimate the number of metals per antibody and label. However, it must be emphasised that this will not deliver accurate molecular weights as the size calibration range of SEC is limited and the separation mechanisms is based on the hydrodynamic radius rather than molecular weight (MW). MW accuracy is further compromised by the logarithmic relationship between retention time and MW. The polymer label detected at $t_{R}=415 \mathrm{~s}$ (Figure 2) corresponds to a theoretical average $\mathrm{MW}$ of $50 \mathrm{kDa}$ which represents an equivalent of approximately 90 monomers per polymer. Figure 3 compares the chromatogram of an unlabelled $(\mathrm{a}), \mathrm{t}_{\mathrm{R}}=381 \mathrm{~s}(\mathrm{MW}=110 \mathrm{kDa})$ ) with a labelled antibody (b), $t_{R}=343 \mathrm{~s}(\mathrm{MW}=230 \mathrm{kDa})$ ). In this case, SEC-ICP-MS underestimated the MW of the unlabelled antibody. The shift in retention time of the labelled and unlabelled antibody corresponded to a calculated molecular mass difference of $\Delta \mathrm{MW}=120 \mathrm{kDa}$ equivalent to an average amount of 2.4 polymer labels per antibody. This value was consistent with data reported by Majonis et al., who used similar chemistries to attach metal loaded polymer labels to antibodies ${ }^{11}$.

Depending on the MW of the labelled antibody and the corresponding protein target, as well as on the linear range of the SEC column, SEC-ICP-MS is capable of characterising immunocomplexes and determination of the protein concentration. Figure $3 \mathrm{c}$ ) shows the chromatographic separation of an immunoassay. One signal was detected at $t_{R}=288 \mathrm{~s}$ (theoretical $M W=690 \mathrm{kDa}$ ) with a shoulder present at $t_{R}=308 \mathrm{~s}$ (theoretical $\mathrm{MW}=460 \mathrm{kDa}$ ) corresponding to an antibody: protein ratio $(\mathrm{APR}) \geq 2: 1$ and equal $1: 1$, respectively. The addition of a secondary antibody (labelled with Dy) resulted in immunocomplexes with APRs of at least 2:1 or higher (Figure $3 \mathrm{~d}$ )). The shoulder at $t_{R}=308 \mathrm{~s}$ (theoretical $\mathrm{MW}=460 \mathrm{kDa}$ ) was not seen and the immunocomplex signal shifted to $t_{R}=281 \mathrm{~s}$. However, due to a lack of separation power in the higher mass range, immunocomplexes with higher APRs cannot be resolved and the abundance of such complexes is solely visible as a widened flattop shaped chromatographic signal. The identified signals are listed in Table 3 together with the respective retention time and the calculated MW.

Figure 4 shows the signals of the labelled lanthanides in an immunoassay with APRs of 2:1 or higher (as shown in Figure $3 \mathrm{~d})$ ). The target protein was directly labelled with ${ }^{153} \mathrm{Eu}$ (blue), the primary antibody with ${ }^{156} \mathrm{Gd}$ (green) and the secondary antibody with ${ }^{163}$ Dy (black). The unconjugated polymer was observed at $t_{R}=415 \mathrm{~s}$ for all three labels. The labelled (unreacted) antibodies and the immunocomplexes were separated and detected at $t_{R}=343 \mathrm{~s}$ and $t_{R}=281 \mathrm{~s}$, respectively.

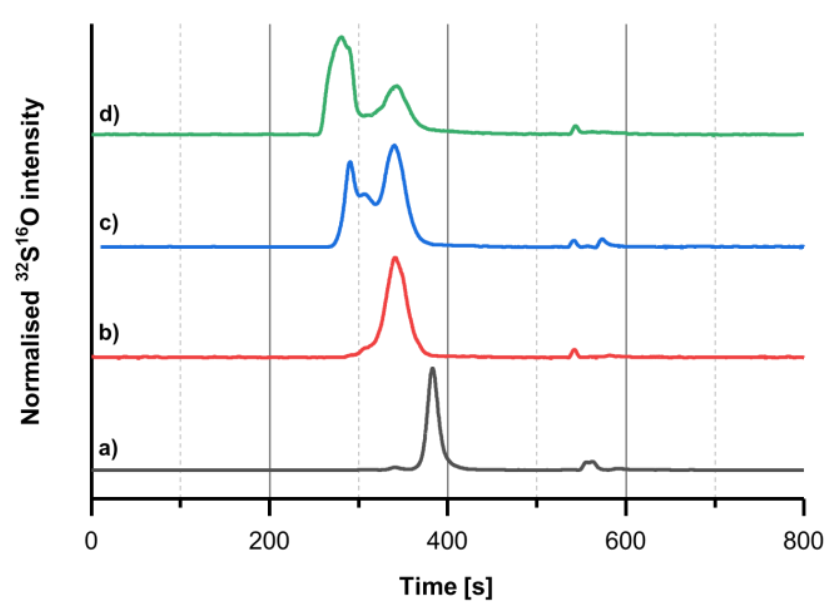

Figure 3. Chromatograms showing the detected sulphur species. a) Unlabelled antibody, b) labelled antibody, c) immunoassay with excess of labelled primary antibody, d) immunocomplexes with APRs of 2:1 and above.

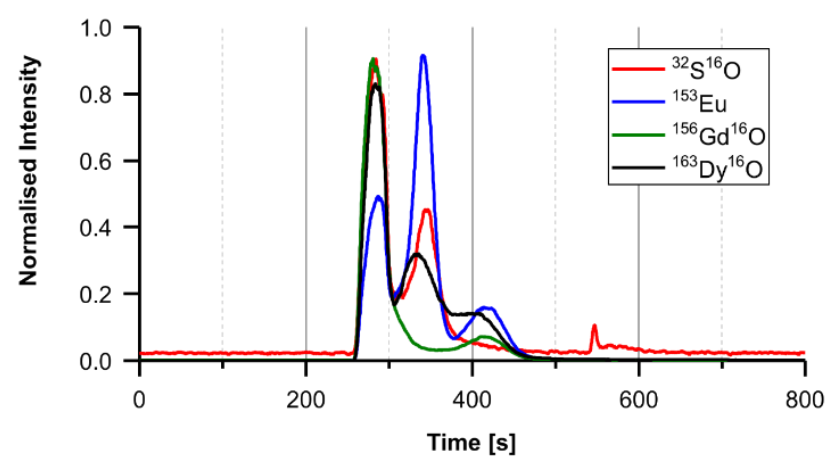

Figure 4. The immunoassay with APRs $\geq 2: 1$ (compare with sulphur chromatogram in Figure $3 \mathrm{~d}$ )). APRs with 1:1 were avoided by spiking an additional labelled (secondary) antibody.

Table 3. Summary of the detected signals. Identification was carried out by performing size calibration using a protein standard for SEC-ICP-MS.

\begin{tabular}{lll}
\hline $\begin{array}{l}\text { Retention } \\
\text { time [s] }\end{array}$ & Identity & $\begin{array}{l}\text { calculated } \\
\text { MW [kDa] }\end{array}$ \\
\hline$>288$ & $\begin{array}{l}\text { Immunocomplexes with } \\
\text { APR ratios > 1:1 }\end{array}$ & 690 \\
308 & $\begin{array}{l}\text { Immunocomplexes with } \\
\text { APR equal 1:1 }\end{array}$ & 460 \\
343 & Labelled antibody & 230 \\
381 & Unlabelled antibody & 110 \\
415 & $\begin{array}{l}\text { Polymer label (unconju- } \\
\text { gated) }\end{array}$ & 50 \\
\hline
\end{tabular}




\section{Quantification of immunoassays with SEC-IDA-ICP-MS}

We have shown that SEC-ICP-MS is a versatile tool to characterise immunoassays qualitatively in terms of estimating MWs of reactants and products and thus, estimating the degree of labelling and antibody: protein stoichiometry. However, SEC does not have the accuracy and precision for a complete characterisation. Absolute quantitative calibration techniques are necessary to accurately determine the degree of labelling as well as the (relative and absolute) quantification of antigens, and therefore the biomolecule.

On-line species unspecific IDA via a post-column spike is a straightforward calibration technique. Quantification of the isotope-enriched labels was performed by reversed on-line IDA approach where a spike containing metals with natural abundance is added post-column to generate a constant isotope ratio. Sulphur was quantified as a marker of the antibody and the biomolecule by conventional on-line IDA with an isotopically enriched S spike added to the post-column eluent. The isotope ratios changed whenever (enriched) lanthanides or sulphur eluted from the SEC column. These ratios were translated into a mass flow $[n g / s]$, and the integration of the mass flow chromatogram allowed calculation of the concentration of unknown peaks. The SEC separations shown in Figure 2 and Figure 4 had the resolution to separate the labelled antibody from the unconjugated polymer, resulting in the direct determination of the ratio of lanthanides and sulphur atoms. However, when the antibody binds to an antigen, the ratio is changed due to sulphur atoms in the target protein. The concentration of the protein is therefore directly determinable from a single chromatogram containing the signal of a labelled antibody and the immunocomplex according to the following equation:

$$
c_{\text {antigen }}\left[\frac{\mathrm{g}}{\mathrm{L}}\right]=\frac{M W_{\text {Protein }} D}{32.065 V_{\mathrm{inj}} N}\left[\int M F_{\mathrm{IC}}^{\mathrm{S}}-\frac{\int M F_{\mathrm{IC}}^{\mathrm{M}} \int M F_{\mathrm{LA}}^{\mathrm{S}}}{\int M F_{\mathrm{LA}}^{\mathrm{M}}}\right]
$$

Where, $\mathrm{c}$ is the concentration, MW the molecular weight, D the dilution factor, $\mathrm{V}_{\text {inj }}$ the injected volume, $\mathrm{N}$ the number of sulphur atoms per protein, MF the mass flow of sulphur (S) or metal (M) of the signal derived from the immunocomplex (IC) or the labelled antibody (LA), respectively. This equation does not require the stoichiometry between protein and antibody to be known for quantification - although the average stoichiometry may be readily calculated. Eliminating the need to know the stoichiometry for quantification allows greater use of polyclonal antibodies in immunoassays that may have multiple antigen sites on the biomolecule, resulting in increased ICP-MS intensity and decreased costs.

Figure 5 shows the mass flow chromatogram of an immunoassay where the protein of interest combined with an ${ }^{153} \mathrm{Eu}-\mathrm{la}$ belled antibody. Sulphur signals detected between $t_{R}=500 \mathrm{~s}$ and $t_{R}=600 \mathrm{~s}$ were identified as impurities. The labelled antibody eluted at $t_{R}=343 \mathrm{~s}$ and integration of the mass flow peak and the application of Equation given above resulted in an average number of $2.39{ }^{153} \mathrm{Eu}$ atoms per sulphur atom. Assuming an average number of 32 sulphur atoms per antibody, this ratio would correspond to an average number of $114 \mathrm{Eu}$ atoms per antibody. These results are consistent with reports describing a similar labelling strategies with 29 and up to 159 lanthanides per antibody ${ }^{11,21}$. As this ratio is contained in the immunocomplex, the increase in sulphur mass of the immunocomplex mass flow peak is directly transferable to the target protein concentration.
This approach determined the protein's sulphur concentration to be $0.0447 \mathrm{nmol} / \mu \mathrm{L}$ corresponding to a protein concentration of $214 \mu \mathrm{g} / \mathrm{mL}$. To validate this approach, the protein was directly labelled with a ${ }^{156} \mathrm{Gd}$ containing polymer $(\mathrm{S}: \mathrm{Gd}=1: 3.81)$ for parallel quantification. This resulted in a protein sulphur concentration of $0.0439 \mu \mathrm{g} / \mathrm{mL}$, corresponding to a total concentration of $210 \mu \mathrm{g} / \mathrm{mL}$ protein. Both approaches were in good agreement with $1.9 \%$ deviation.

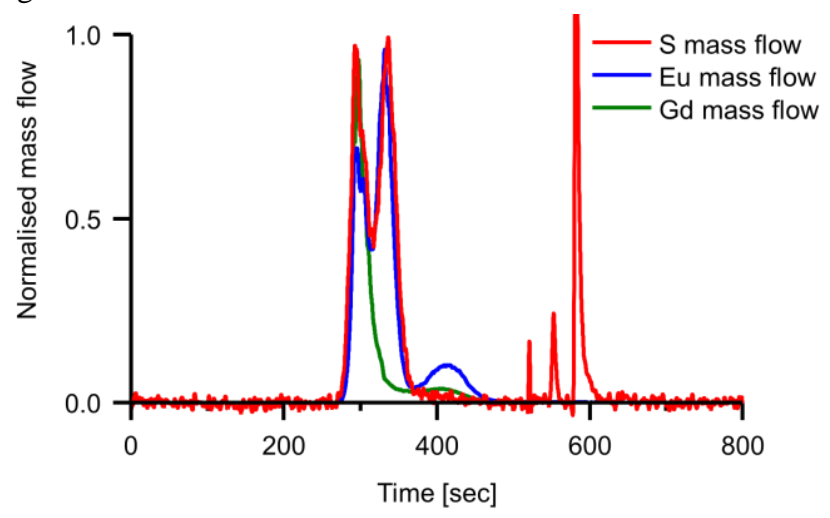

Figure 5. The mass flow chromatograms of S, Eu and Gd of an immunoassay where the protein of interest $(\mathrm{IgG})$ was directly labelled with ${ }^{156} \mathrm{Gd}$, and then reacted with an ${ }^{153}$ Eu-labelled polyclonal antibody.

\section{CONCLUSION}

This work presented novel methods for the direct and rapid analysis of labelled antibodies and immunoassays to ensure integrity of labelled antibodies and to determine the stoichiometry between metals, labels, antibodies and biomolecules. Massshifting via the addition of oxygen into the DRC allowed the analysis of sulphur and lanthanide signals with high sensitivity and low background. To avoid interferences, analyses were performed without antibody stabiliser that potentially contained sulphur-based compounds. SEC-ICP-MS was used to demonstrate changes occurring to labelled antibodies during storage and to develop a chromatographic separation of labelled and unlabelled antibodies, immunocomplexes and the unconjugated lanthanide loaded polymer. To improve the accuracy in determining the antibody/protein stoichiometry and to quantify the biomolecule, a novel on-line SEC-IDA-ICP-MS method was developed which allowed the absolute quantification of an $\mathrm{IgG}$ protein reacted with a polyclonal antibody. The chromatographic separation of the excess labelled-antibody and the immunocomplex peaks resulted in the determination of the metal: sulphur stoichiometry of the labelled antibody. Secondly, the differences in sulphur between the antibody and the immunocomplex peak was accounted for and used to calculate the concentration of the biomolecule. 


\section{Corresponding Author}

* Philip Doble, e-mail: Philip.Doble@uts.edu.au, Telephone: $+\mathbf{6 1 2 9 5 1 4 1 7 9 2}$

\section{Author Contributions}

The manuscript was written through contributions of all authors. I All authors have given approval to the final version of the manuscript. / \$These authors contributed equally.

\section{ACKNOWLEDGMENT}

D.C. is funded by the Deutsche Forschungsgemeinschaft (DFG. German Research Foundation) - 417283954.

DPB is supported by an Australian Research Council Discovery Early Career Researcher Award (DE180100194). PAD is the recipient of an Australian Research Council Discovery Project (DP170100036).

\section{REFERENCES}

(1) Becker, J. S.; Jakubowski, N. Chem. Soc. Rev. 2009, 38 (7), 1969-1983.

(2) Mounicou, S.; Szpunar, J.; Lobinski, R. Chem. Soc. Rev. 2009, 38, 1119-1138.

(3) Peng, W.; Ling-Ni, M.; He-Fang, W.; Xue-Guang, S.; Xiu-Ping, Y. Angew. Chemie 2011, 123 (35), 8268-8271.

(4) Biju, V.; Itoh, T.; Ishikawa, M. Chem. Soc. Rev. 2010, 39 (8), 3031-3056.

(5) Yalow, R. S.; Berson, S. A. Nature 1959, 184, 1648.

(6) Zhang, C.; Wu, F.; Zhang, Y.; Wang, X.; Zhang, X. J. Anal. At. Spectrom. 2001, 16 (12), 1393-1396.

(7) Mairinger, T.; Wozniak-Knopp, G.; Rüker, F.; Koellensperger, G.; Hann, S. J. Anal. At. Spectrom. 2016, 31 (11), 2330-2337.

(8) Esteban-Fernández, D.; Scheler, C.; Linscheid, M. W. Anal Bioanal. Chem. 2011, 401 (2), 657-666.

(9) Giesen, C.; Wang, H. A.; Schapiro, D.; Zivanovic, N.; Jacobs, A.; Hattendorf, B.; Schüffler, P. J.; Grolimund, D.; Buhmann, J. M.;
Brandt, S.; Varga, Z.; Wild, P. J.; Günther, D.; Bodenmiller, B. Nat. Methods 2014, 11, 417.

Majonis, D.; Ornatsky, O.; Kinach, R.; Winnik, M. A. Biomacromolecules 2011, 12 (11), 3997-4010.

(11) Majonis, D.; Herrera, I.; Ornatsky, O.; Schulze, M.; Lou, X; Soleimani, M.; Nitz, M.; Winnik, M. A. Anal. Chem. 2010, 82 (21), 8961-8969.

(12) Lou, X.; Zhang, G.; Herrera, I.; Kinach, R.; Ornatsky, O.; Baranov, V.; Nitz, M.; Winnik, M. A. Angew. Chemie - Int. Ed. 2007, 46 (32), 6111-6114.

(13) Angelo, R. M. Method for multiplexed sample analysis by photoionizing secondary sputtered neutrals. Patent Number CA2981496A1, 2015.

(14) Schwarz, G.; Mueller, L.; Beck, S.; Linscheid, M. W. J. Anal. At. Spectrom. 2014, 29 (2), 221-233.

(15) He, Y.; Esteban-Fernandez, D.; Neumann, B.; Bergmann, U.; Bierkandt, F.; Linscheid, M. W. J. Proteomics 2016, 136, 68-76. Hann, S.; Boeck, K.; Koellensperger, G. 2010, 25 (1), 18-20.

(17) Hempen, C.; Karst, U. Anal. Bioanal. Chem. 2006, 384 (3), 572 583.

(18) Terenghi, M.; Elviri, L.; Careri, M.; Mangia, A.; Lobinski, R. Anal. Chem. 2009, 81 (22), 9440-9448.

(19) Campanella, B.; Bramanti, E. Analyst 2014, 139 (17), 4124-4153.

(20) Mueller, L.; Mairinger, T.; Hermann, G.; Koellensperger, G.; Hann, S. Anal. Bioanal. Chem. 2014, 406 (1), 163-169.

(21) Pérez, E.; Bierla, K.; Grindlay, G.; Szpunar, J.; Mora, J.; Lobinski, R. Anal. Chim. Acta 2018, 1018, 7-15.

(22) Benda, D.; Schwarz, G.; Beck, S.; Linscheid, M. W. J. Mass Spectrom. 2014, 49 (1), 13-18.

(23) Quinn, T. J. Metrologia 1997, 34 (1), 61.

(24) Rodriguez-Gonzalez, P.; Marchante-Gayon, J. M.; Garcia Alonso, J. I.; Sanz-Medel, A. Spectrochim. Acta - Part B At. Spectrosc. 2005, 60 (2), 151-207.

(25) Yan, X.; Xu, M.; Yang, L.; Qiuquan, W. Anal. Chem. 2010, 82 (4), 1261-1269.

(26) Kretschy, D.; Koellensperger, G.; Hann, S. Anal. Chim. Acta 2012, 750, 98-110.

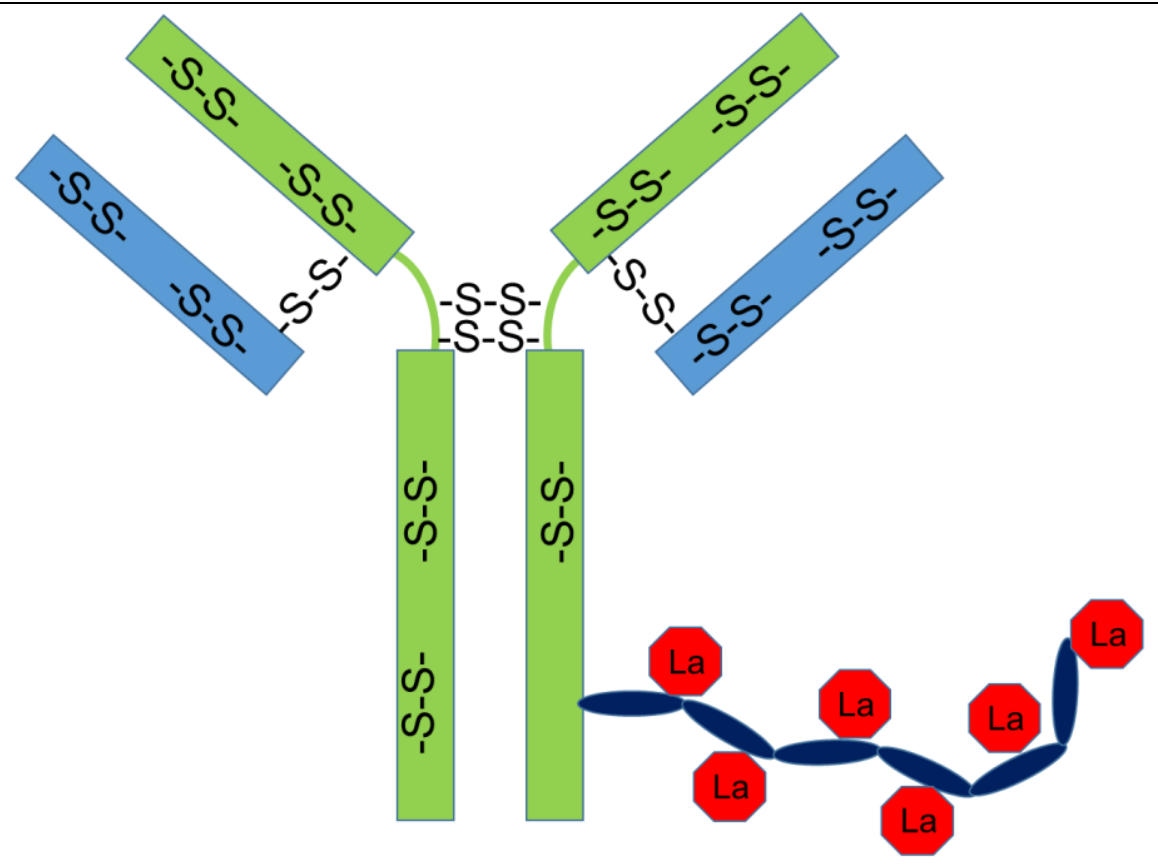

Table of Contents artwork 